\title{
Chronic use of benzodiazepines and psychomotor and cognitive test performance
}

\author{
Irwin Lucki, Karl Rickels, and Andrew M. Geller* \\ Department of Psychiatry, University of Pennsylvania School of Medicine, Philadelphia, PA 19104, USA
}

\begin{abstract}
The performance of 43 long-term users (average $=$ 5 years) of benzodiazepine (BZ) medications was examined on a battery of behavioral tasks, cognitive tests, and subjective mood rating scales. The performance of the chronic BZ users did not differ significantly from age- and sexmatched anxious subjects, except that critical flicker fusion (CFF) thresholds were lower and subjective ratings of tranquilization were higher in the BZ users. Twenty-two subjects were reexamined in order to determine the acute effects of $\mathrm{BZ}$ medications in long-term users. The acute administration of BZ medications significantly increased CFF thresholds, improved digit-symbol substitution test performance, impaired the delayed recall of verbal material, increased subjective ratings of tranquilization, and reduced physical sedation. Motor performance tests were not impaired and subjective feelings of sedation were not increased after the acute administration of BZs by chronic users. During withdrawal from long-term BZ use (17 subjects), CFF thresholds were elevated, subjective ratings of physical sedation and anxiety were increased, but performance on other psychomotor and cognitive tests was not altered. The results suggest that tolerance develops selectively to different behavioral and subjective effects of BZ medications with their continued use. Tolerance failed to develop to the antianxiety effects, the reduction of CFF threshold, and to the impairment of short-term memory caused by BZs. However, chronic users of $\mathrm{BZ}$ medications failed to demonstrate psychomotor-impairing or sedating effects to $\mathrm{BZ}$ medications. The results have implications for evaluating the safety of the long-term use of $\mathrm{BZ}$ medications.
\end{abstract}

Key words: Benzodiazepines - Chronic drug use - Anxiety Psychomotor performance - Memory

A number of benzodiazepine (BZ) derivatives have become widely used in medical and psychiatric practice due to their effectiveness as antianxiety agents, hypnotics, muscle relaxants, and in the control of seizures (Rickels 1978; Cohen 1981). There has long been concern about psychological side effects that might be produced by these drugs. The

* Present address: Department of Psychology, University of Michigan, Ann Arbor, MI 48104, USA

Offprint requests to: I. Lucki, Psychopharmacology Research and Treatment Clinic, 133 South 36th St. Room 402, Philadelphia, PA 19104 USA initial administration of $\mathrm{BZs}$ to normal volunteers produces sedation and impairment on a variety of psychomotor tasks that measure motor speed, visuomotor coordination, and cognitive function (see Vogel 1979; Wittenborn 1979; Kleinknecht and Donaldson 1975). Similar effects have been reported with anxious subjects (Linnoila et al. 1983; de Gier et al. 1981; Zimmerman-Tansella et al. 1978), so that patients should be cautioned about driving or operating dangerous machinery when taking these medications. However, these concerns can be decreased by physicians when therapy is initiated at drug doses with minimal sedating effects (Rickels 1978, 1981). Furthermore, because the sedative effects of $\mathrm{BZs}$ appear to diminish rapidly with repeated administration while their antianxiety effects remain effective (Rickels et al. 1983), the therapeutic dosage of BZ medications can be adjusted safely during their continued administration.

Although BZs are most often taken regularly by patients for relatively short periods of time, many individuals, particularly the elderly, may take them chronically for years (Mellinger and Balter 1981). There has been growing concern recently about deleterious effects that may be associated with the long-term use of BZ medications. Patients who have taken BZs regularly for long periods of time may experience withdrawal symptoms following abruptly stopping the drug, even if they have been taking medicallyprescribed doses (Winokur and Rickels 1981; Winokur et al. 1980). These patients can be managed by gradually reducing their dose. Of greater concern are reports of patients taking $\mathrm{BZs}$ regularly for years that demonstrate signs of generalized intellectual impairment (Hendler et al. 1980; Bergman et al. 1980). Recently, Petursson et al. (1983) reported impaired performance by long-term BZ users on the digit-symbol substitution test and the symbol copying test, although performance on tests of reaction time or letter cancellation was not altered. Furthermore, the impaired performance of these patients failed to recover after they gradually stopped taking their medication. With the notable exception of Petursson et al. (1983), the effects of BZs on objective performance tests have not been studied in patients that have taken these medications longer than for just a few weeks.

The present study was conducted as part of a clinical program examining the medical and psychological status of patients who have taken medically-prescribed doses of $\mathrm{BZ}$ medications for long periods of time. Forty-three patients taking BZs daily for an average of 5 years were exam- 
ined at their initial clinic visit on a battery of psychomotor tests, cognitive tasks, and subjective mood rating scales. These tests were selected because they had been shown previously to be sensitive to detecting the effects of BZs in normal volunteers (Vogel 1979; Wittenborn 1979; Kleinknecht and Donaldson 1975). Their test scores were compared with a matched group of drug-free anxious outpatients. Twenty-two of the chronic users returned to the clinic when the acute effects of the BZ medications were examined shortly after their medication was taken. Finally, changes in performance were examined in 17 subjects during the time that they were experiencing symptoms of withdrawal from BZs. A preliminary report of these data appeared earlier (Lucki et al. 1985).

\section{Materials and methods}

\section{Subjects}

The chronic BZ users $(N=43)$ were recruited from the BZ Discontinuation Program conducted by the Psychopharmacology Research and Treatment Clinic at the University of Pennsylvania. The patients were 22 males and 21 females with an average age of 43.5 years ( \pm 1.7 years, 1 SEM; range $=26-70)$. All of the patients, except for six $(14 \%)$, graduated high school and 12 of the patients $(28 \%)$ were college graduates. All 43 patients were taking a variety of BZs at the time of their initial visit. Thirteen patients were taking diazepam (Valium, mean $=11.1 \mathrm{mg} /$ day, range $=5$ $20 \mathrm{mg} /$ day), 13 patients were using lorazepam (Ativan, $3.7 \mathrm{mg} /$ day, $1-10 \mathrm{mg} /$ day), seven patients were receiving clorazepate dipotassium (Tranxene, $10.0 \mathrm{mg} /$ day, $3.75-15 \mathrm{mg} /$ day), and seven patients were using alprazolam (Xanax, $2.6 \mathrm{mg} /$ day, $0.75-6 \mathrm{mg} /$ day). In nearly every case, the daily intake of $\mathrm{BZ}$ medication was within the range acceptable for therapeutic use. As a group, the patients had been taking their BZ medications regularly (defined as daily intake) for an average of 60 months ( \pm 1.2 months; range $=11-185$ ). The range of the duration of $B Z$ use varied with the patient's primary medication: diazepam, 14 185 months; lorazepam, 15-120 months; clorazepate, 1184 months; and alprazolam, 14-23 months. Individual patients were also examined that were taking either chlordiazepoxide (Librium, $40 \mathrm{mg} /$ day for 120 months), oxazepam (Serax, $10 \mathrm{mg} /$ day for 36 months), or flurazepam (Dalmane, $30 \mathrm{mg}$ /day for 108 months). Of the 43 patients, 33 $(77 \%)$ were taking only a single $\mathrm{BZ}$ as their medication. Four patients were simultaneously taking two BZs; flurazepam (2) and triazolam (1) were taken occasionally at night. for sleep and one patient was regularly taking clorazepate and lorazepam. Six patients were concomitantly taking nonbenzodiazepine medications; four instances involved the additional use of an antidepressant drug and two patients were taking antihypertension medications. The behavior of these six subjects did not differ systematically from the remaining group. A 5-ml sample of blood was taken at the initial interview and examined for the presence of $\mathrm{BZ}$ medications or metabolites in plasma.

The psychiatric interviews at the initial evaluation revealed the existence of psychiatric problems in many of the chronic users of BZs. Most of the patients were diagnosed with an anxiety-related disorder; $24(56 \%)$ with generalized anxiety disorder and $13(30 \%)$ with panic disorder. Of the remaining patients, two were diagnosed with major depressive disorder, two had a cyclothymic disorder, and two patients had symptoms of mixed anxiety and depression.

A group of controls $(N=26)$ was recruited from patients seeking treatment for anxiety-related disorders at the Clinic. This group of subjects was matched for sex (15 males, $11 \mathrm{fe}-$ males), educational level, and age (mean $=38.6$ years, \pm 2.2 , $P>0.05$ ) with the group of chronic BZ patients. Each of these control subjects was diagnosed with Generalized Anxiety Disorder (DSM III criteria) after a psychiatric interview and each patient scored greater than 18 on the Hamilton Anxiety Scale. All of the control subjects were drug-free for at least 3 weeks prior to taking the battery of behavioral tests and none had been chronic users of $\mathrm{BZ}$ medications.

\section{Behavior test battery}

A battery of six tests were administered at each session. The tests are described briefly below in the order of their administration. The behavioral tests were selected because they were previously reported to be sensitive to the effects of BZs in normal volunteers (see Vogel 1979; Wittenborn 1979; Kleinknecht and Donaldson 1975).

Subjective mood scales. The subjective report of mood states was measured using $100-\mathrm{mm}$ lines that separated 12 pairs of items that described opposite feeling states, as described by Norris (1971). Scales of mental sedation (alert/drowsy, clear headed/fuzzy, quick witted/mentally slow, attentive/ dreamy), physical sedation (strong/feeble, well coordinated/ fuzzy, energetic/lazy, capable/incompetent) and tranquilization (calm/excited, contented/discontented, peaceful/agitated, relaxed/tense) were constructed by grouping the scores from four similar items. A larger score indicates a report of more intense feelings of mental sedation, physical sedation, or tranquilization.

Digit-symbol substitution test (DSST). A code of nine matched digits and symbols was presented at the top of the test form. The DSST required subjects to record the appropriate symbol below the sample digits that were matched by a code appearing at the top of the form. The test was scored as the number of correct items completed in $90 \mathrm{~s}$. Different forms and codes were used each time that the test was administered in order to prevent subjects from improving their performance by memorizing a single code. This test was similar to one of the subtests of the Wechsler Adult Intelligence Scale (WAIS, Wechsler 1955). The DSST and the symbol copying test were readministered at the end of the initial interview because patients rapidly became familiar with the testing procedure, and the score on the second administration was used.

Symbol copying test. The symbol copying test required subjects to draw symbols immediately below a sample. The nine same symbols that were used in the DSST were presented in a random order on each test form. Different forms were used each time that the test was given. The test was scored as the number of items completed in $90 \mathrm{~s}$. This test has been used, in combination with the DSST, as a motoric control for physical writing speed (Petursson et al. 1983).

Letter cancellation test. The letter cancellation test required subjects to scan a form of randomly-arranged letters and 
to circle a given target letter. Sixty seconds were allowed for this test. Items scored were the number of letters scanned and the number of times that the target letter appeared but was not circled (scored as an omission).

Critical flicker fusion threshold (CFF). The CFF threshold was included in the test battery because it has been shown to be sensitive to the effects of psychoactive medications including BZs (Smith and Misiak 1976), although its significance is not completely understood. The CFF was measured using a Leeds Psychomotor Tester apparatus. Subjects examined an illuminated display of four red light-emitting diodes (LEDs), placed $1 \mathrm{~m}$ from the subject at approximately eye level, that was continuously changing in frequency. The subjects pressed an indicator button when they observed the display of flickering lights change to appear continuously illuminated (trials of ascending frequency) or when the continuously illuminated display changed to appear to flicker (trials of descending frequency). Trials of ascending and descending frequency were presented alternately, with the first two trials administered for practice but not scored. The score was the combined average frequency of five trials of ascending frequency and five trials of descending frequency.

Free recall of word lists. Verbal memory was measured after reading a list of 16 noncategorized nouns to subjects at a rate of one word/ $2 \mathrm{~s}$. The subjects recorded as many of the words that they could recall immediately after the list of words was read, and then recorded as many words from the same list that they could still remember 20 min later. Immediate and delayed recall were measured as the absolute number of words remembered after no delay and after a 20 -min delay. In addition, the memory decrement was measured as the percentage of words that were remembered immediately that could no longer be recalled $20 \mathrm{~min}$ later [1-(delayed recall/immediate recall)*100]. Separate lists were used for each presentation composed of nouns that were matched for their relative frequency of use in the English language (Thorndike and Lorge 1944; Paivio et al. 1968).

\section{Testing procedure}

The initial behavior evaluation of 43 chronic BZ subjects occurred during their initial visit to the Psychopharmacology Research and Treatment Clinic. The subjects were seen at various times of the day without regard to the time of their previous dose of medication. The subjects were told not to take their medication just prior to arrival at the Clinic, so that the behavior tests were conducted approximately $4-14 \mathrm{~h}$ after their previous dose of medication. Thus, this evaluation might be regarded as representing the status of these patients as they ordinarily appear in their daily activities. The performance of the 43 chronically-medicated subjects at this evaluation was compared with the group of 26 drug-free anxious subjects.

Twenty-two of the chronic BZ subjects returned to the clinic 3 weeks after their initial visit to assess the acute effect of their BZ medications. The patients that participated in this lengthier test session did not differ systematically from the larger group of chronic BZ users but were selected on the basis of their availability. Of this subset of subjects, nine were taking diazepam, six were taking lorazepam, four subjects were using clorazepate, two were receiving alpra- zolam, and one subject was taking chlordiazepoxide. They were instructed to eat only a light breakfast (juice and no caffeinated beverages) and to refrain from taking any medication immediately prior to their visit. Upon their arrival in the laboratory, the patients were tested on the battery of behavior tests. The subjects then took a fixed dose of their medication (often their usual unit dose) and were retested on the behavior test battery starting 60-90 min later, or during the time of expected peak drug effects. The dose of drug administered to these subjects was assigned according to their regular medication: $5 \mathrm{mg}$ diazepam, $1 \mathrm{mg}$ lorazepam, $7.5 \mathrm{mg}$ clorazepate, $0.5 \mathrm{mg}$ alprazolam, or $10 \mathrm{mg}$ chlordiazepoxide. Their performance following medication was compared with their test scores measured immediately prior to their taking the drug.

Many of the chronically-medicated subjects abruptly stopped taking their medication as part of a supervised drug withdrawal program. It was possible to readminister the behavior test battery to some of these subjects $(N=17)$ during a subsequent visit to the Psychopharmacology Clinic, approximately 4-8 days following the cessation of their BZ intake. The time of this evaluation varied according to the individual clinical condition of the subjects. Most of the subjects reported experiencing some withdrawal symptoms at this evaluation and these clinical data will be reported separately at a later time. The 17 subjects that participated in this evaluation were regularly taking the following BZ medications: diazepam (7), clorazepate (4), lorazepam (3), and one subject each taking alprazolam, oxazepam, or chlordiazepoxide. The behavioral performance of these subjects was compared with their own performance determined on the day of their initial visit to the Psychopharmacology Clinic.

\section{Results}

\section{Initial evaluation of chronic $B Z$ users}

Analysis of the plasma samples from the chronically-medicated subjects confirmed the presence of high concentrations of BZ medications or metabolites for all subjects. Mean plasma levels $( \pm 1$ SEM) of the following drugs or metabolites were measured: 1) diazepam, $517 \pm 108 \mathrm{ng} / \mathrm{ml}$, plus desmethyldiazepam (active metabolite), $582 \pm 135 \mathrm{ng} /$ $\mathrm{ml}$; 2) clorazepate (not measured) or desmethyldiazepam (active metabolite), $360 \pm 101 \mathrm{ng} / \mathrm{ml}$; 3) lorazepam, $38 \pm 6 \mathrm{ng} / \mathrm{ml}$; and 4) alprazolam, $30 \pm 9 \mathrm{ng} / \mathrm{ml}$.

The assessment of behavioral performance of the 43 subjects who had taken BZ medications for years did not reveal strong evidence of changes when compared with the group of drug-free anxious controls with a few exceptions (see Table 1). Test performance by the chronic BZ group did not differ significantly on the DSST, symbol copying test, letter cancellation test, or verbal recall tests. The CFF threshold showed a small $(4.5 \%)$, but statistically significant $(P<0.05)$, reduction in the chronic BZ subjects when compared with the anxious controls. The subjective report of physical or mental sedation did not differ between groups, but the chronic BZ subjects did report a greater magnitude of response on the tranquilization scale $(P<$ 0.05 ) than the drug-free anxious subjects.

The initial behavioral test performance was examined separately in those subjects taking BZs with long half-lives (e.g., diazepam, clorazepate, $N=20$ ) versus the $\mathrm{BZs}$ with 
Table 1. Behavioral performance of chronic users of benzodiazepine medications

\begin{tabular}{|c|c|c|c|c|}
\hline Behavior test & $\begin{array}{l}\text { Drug-free } \\
\text { anxious } \\
\text { controls }\end{array}$ & $N$ & $\begin{array}{l}\text { Chronic } \\
\text { BZ patients }\end{array}$ & $N$ \\
\hline $\begin{array}{l}\text { Digit-symbol } \\
\text { substitution test }\end{array}$ & $62.6 \pm 2.7$ & 26 & $59.3 \pm 1.7$ & 42 \\
\hline $\begin{array}{l}\text { Symbol copying } \\
\text { test }\end{array}$ & $144.3 \pm 5.1$ & 26 & $136.8 \pm 3.9$ & 42 \\
\hline \multicolumn{5}{|l|}{ Letter cancellation test } \\
\hline $\begin{array}{l}\text { Number of letters } \\
\text { Omissions }\end{array}$ & $\begin{array}{r}495.9 \pm 22.4 \\
1.5 \pm 0.4\end{array}$ & 26 & $\begin{array}{r}462.0 \pm 16.6 \\
1.3 \pm 0.2\end{array}$ & 42 \\
\hline $\begin{array}{l}\text { Critical flicker } \\
\text { fusion threshold } \\
(\mathrm{Hz})\end{array}$ & $32.46 \pm 0.52$ & 24 & $30.99 \pm 0.40^{*}$ & 42 \\
\hline \multicolumn{5}{|l|}{ Free verbal recall } \\
\hline $\begin{array}{l}\text { Immediate recall } \\
\text { Delayed recall } \\
\text { Memory decrement } \\
(\%)\end{array}$ & $\begin{array}{r}9.0 \pm 0.6 \\
7.0 \pm 0.6 \\
23.5 \pm 3.9\end{array}$ & 22 & $\begin{array}{r}7.9 \pm 0.3 \\
5.8 \pm 0.4 \\
28.1 \pm 3.0\end{array}$ & 40 \\
\hline \multicolumn{5}{|l|}{ Mood rating scales } \\
\hline $\begin{array}{l}\text { Mental sedation } \\
\text { Physical sedation } \\
\text { Tranquilization }\end{array}$ & $\begin{array}{l}175.8 \pm 15.9 \\
150.8 \pm 11.2 \\
186.4 \pm 11.6\end{array}$ & 22 & $\begin{array}{l}147.5 \pm 12.9 \\
131.2 \pm 13.0 \\
234.3 \pm 16.2^{*}\end{array}$ & 39 \\
\hline
\end{tabular}

All values represent the mean \pm 1 SEM

* Value differs significantly from drug-free anxious controls according to Student's $t$-test, $P<0.05$

relatively short half-lives (e.g., lorazepam, alprazolam, $N=$ 20 ) and is shown in Table 2 . No significant differences were measured in performance on any of the psychomotor tests, verbal recall tests, or report of subjective mood between these two broad classes of BZ medications $(P>0.05$ for all comparisons).

\section{Acute effect of BZs in chronic drug users}

The acute effects of a variety of BZs were examined in 22 of the subjects 3 weeks after their initial evaluation. The predrug test performance of these subjects did not differ significantly from their test scores measured at their initial interview. The results for most of the behavior tests are presented in Table 3. Performance on the DSST showed a small but statistically significant improvement $(P=0.02)$. The $\mathrm{CFF}$ threshold demonstrated a significant reduction shortly after intake of drug in the chronic BZ subjects. Test scores for symbol copying and letter cancellation did not differ from predrug values.

The acute administration of the BZs produced interesting effects on the verbal recall tests (see Fig. 1). Although immediate recall was not altered, the ability to recall the word list after a 20 -min delay was significantly impaired $(P=0.005)$ shortly after drug intake. This effect was also reflected in a significant increase in memory decay, or the percentage of words that were remembered immediately that could not be recalled after the 20 -min delay.

The acute intake of BZ medication did not change the reported level of mental sedation (see Fig. 2), but decreased significantly $(P=0.01)$ feelings of physical sedation in the
Table 2. Comparison of benzodiazepines with different half-lives on behavioral performance

\begin{tabular}{lrr}
\hline Behavior tests & $\begin{array}{c}\text { Short-acting } \\
\text { benzodiazepines }\end{array}$ & $\begin{array}{c}\text { Long-acting } \\
\text { benzodiazepines }\end{array}$ \\
\hline $\begin{array}{l}\text { Digit-symbol substitution } \\
\text { Symbol copying test }\end{array}$ & $60.3 \pm 2.3$ & $57.6 \pm 2.4$ \\
Letter cancellation test & $142.6 \pm 5.6$ & $131.0 \pm 5.6$ \\
$\quad$ Number of letters & $465.1 \pm 25.0$ & $455.5 \pm 25.3$ \\
$\quad$ Omissions & $1.4 \pm 0.3$ & $1.2 \pm 0.4$ \\
Critical flicker fusion & $30.77 \pm 0.57$ & $31.20 \pm 0.64$ \\
theshold (Hz) & & \\
Free verbal recall & $7.6 \pm 0.5$ & $8.2 \pm 0.5$ \\
$\quad$ Immediate recall & $5.6 \pm 0.6$ & $6.0 \pm 0.5$ \\
$\quad \begin{array}{l}\text { Delayed recall } \\
\text { Memory decrement }(\%)\end{array}$ & $27.5 \pm 5.0$ & $27.9 \pm 3.7$ \\
Mood rating scales & & \\
$\quad$ Mental sedation & $145.1 \pm 17.4$ & $142.6 \pm 21.4$ \\
$\quad$ Physical sedation & $127.8 \pm 17.4$ & $134.5 \pm 24.5$ \\
$\quad$ Tranquilization & $253.2 \pm 22.3$ & $204.3 \pm 27.3$ \\
\hline
\end{tabular}

All values represent the mean \pm 1 SEM for patients taking either short-acting (i.e., lorazepam or alprazolam; $N=20$ ) or long-acting (i.e., diazepam or clorazepate; $N=20$ ) benzodiazepines. All comparisons between the two classes of benzodiazepines were not statistically significant $(P>0.05)$, according to Student's $t$-test

Table 3. Effect of acute administration of benzodiazepines on the behavioral performance of chronically medicated patients

\begin{tabular}{lrr}
\hline Behavior test & \multicolumn{1}{c}{ Predrug } & \multicolumn{1}{c}{ Postdrug } \\
\hline Digit-symbol substitution & $56.9 \pm 2.5$ & $59.4 \pm 2.8^{*}$ \\
Symbol copying test & $137.4 \pm 4.6$ & $141.0 \pm 5.2$ \\
Letter cancellation test & & \\
$\quad$ Number of letters & $483.3 \pm 22.9$ & $482.8 \pm 23.5$ \\
$\quad$ Omissions & $1.0 \pm 0.3$ & $1.3 \pm 0.3$ \\
$\begin{array}{l}\text { Critical flicker fusion } \\
\text { threshold (Hz) }\end{array}$ & $32.32 \pm 0.66$ & $31.71 \pm 0.69^{*}$ \\
\hline
\end{tabular}

All values represent the mean \pm 1 SEM

* Value differs significantly from predrug performance according to Student's $t$-test, $P<0.05$

chronic $\mathrm{BZ}$ patients. In addition, acute intake of $\mathrm{BZs}$ increased significantly $(P=0.001)$ the report of subjective feelings of tranquilization.

Although the sample of subjects examined with some of the drugs was small, it appeared that the pooled results were representative of the effect for each medication. On the verbal recall test, for example, the drugs with a long half-life $(N=13)$ produced as large a reduction in delayed recall $(5.0$ words \pm 0.7$)$ and memory decay $(44.5 \% \pm 6.2)$ as the $\mathrm{BZ}$ medications with a short half-life ( 4.9 words \pm 1.0 and $38.8 \% \pm 7.1$, respectively). Similarly, the magnitude of subjective tranquilization was increased equivalently by the long-acting $(255 \pm 27)$ as well as the short-acting $(245 \pm 32)$ BZs.

\section{Behavior test performance during $B Z$ withdrawal}

Seventeen subjects were examined with the behavior test battery 4-8 days after they stopped taking their BZ medica- 


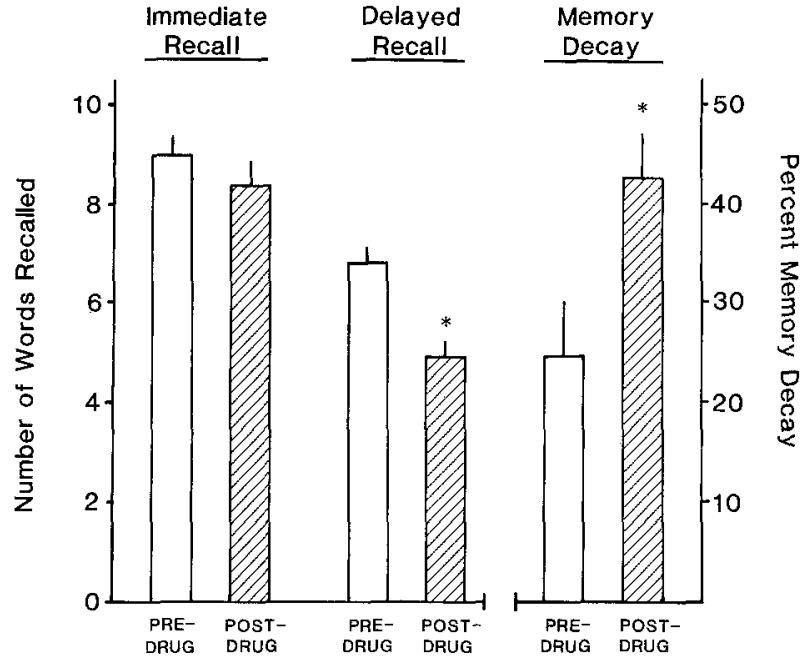

Fig. 1. Effect of acute administration of $B Z s$ on the free recall of word lists in long-term BZ users. The values shown on the left side of the figure represent the mean number of words recalled, with vertical lines showing 1 SEM. The values shown on the right side of the figure represent the percent decay of memory after 20 min relative to the immediate recall of the word list (1-delayed recall/immediate recall). The $\mathrm{BZs}$ did not alter immediate recall $(P>0.05)$ but significantly reduced the number of words recalled after a 20 -min delay $[t(19)=3.13, P=0.005]$. In addition, the memory decay percentage was also significantly increased by acute $\mathrm{BZ}$ administration in long-term $\mathrm{BZ}$ users $[t(19)=2.74, P=0.01]$

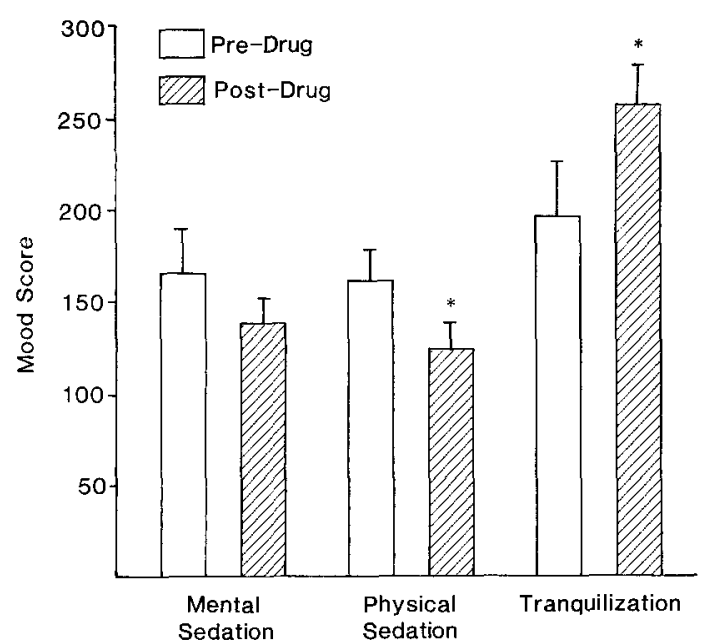

Fig. 2. Alterations in mood state caused by the acute administration of BZ medications to long-term users. The values shown represent the mean mood score on the scales of mental sedation, physical sedation, or tranquilization before or $1 \mathrm{~h}$ after taking $\mathrm{BZ}$ medications, with the vertical lines showing 1 SEM. Acute administration of BZs produced a significant reduction in feelings of physical sedation and increased feelings of tranquilization (less anxiety) in the chronic users. ${ }^{*}$ indicates $P=0.01$ according to Student's $t$-test

tions. The behavior test performance of these subjects is presented in Table 4. These subjects showed no significant differences in their performance on the DSST, symbol copying, letter cancellation, and verbal recall tasks when compared with their own performance at their initial interview. However, the CFF thresholds of the chronic BZ subjects increased significantly $(P=0.004)$ during $\mathrm{BZ}$ withdrawal (see Table 4). In addition, as shown in Fig. 3, the chronic
Table 4. Changes in behavioral performance of chronic benzodiazepine users following abrupt withdrawal from medication

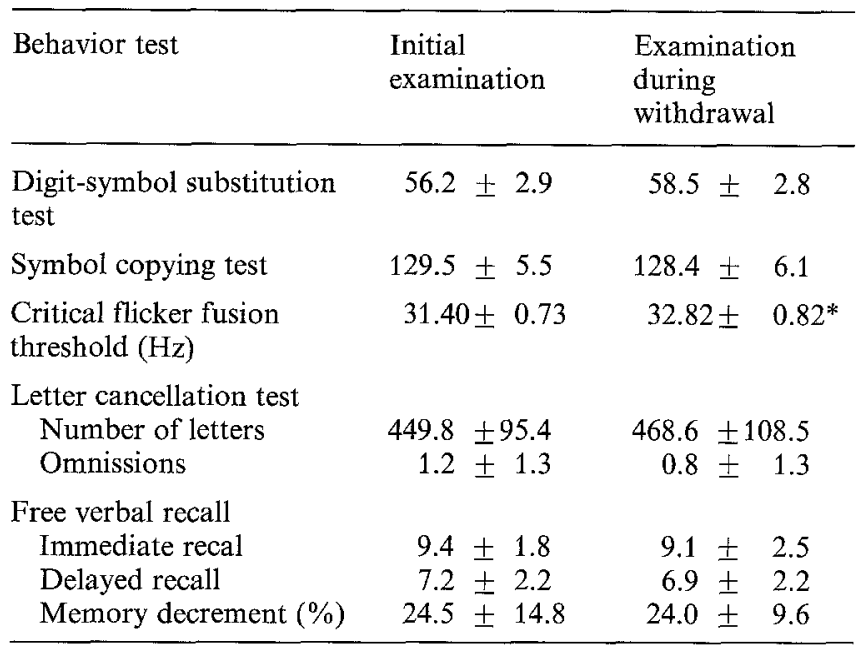

The initial examination of chronic benzodiazepine users $(N=17)$ was performed during the patient's first visit to the clinic. The examination during drug withdrawal occurred 4-5 weeks later and approximately 4-8 days after the patients stopped taking their medication. All values represent the mean \pm 1 SEM

* Value is significantly higher than performance during the initial examination according to Student's $t$-test, $t(15)=3.37, P=0.004$

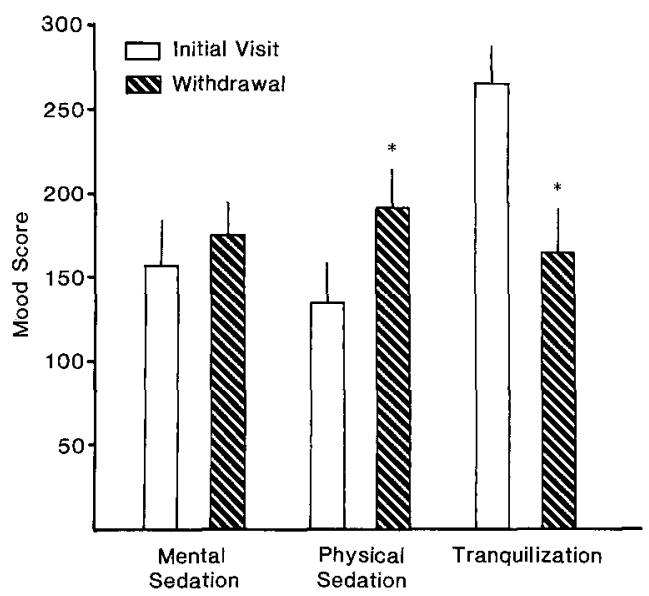

Fig. 3. Changes in mood state during withdrawal from long-term use of BZ medications. Patients were evaluated 4-8 days after stopping their medication and their scores were compared with the report of their mood state during the initial interview (when they were on medication). Patients reported significantly greater feelings of physical sedation and reduced feelings of tranquilization (greater anxiety) during the period of withdrawal from BZs. * indicates that the value differs significantly from baseline according to Student's $t$-test, $P<0.05$

BZ subjects reported an increase in their subjective feeling of physical sedation $(P=0.03)$ and a reduction in their reported feeling of tranquilization $(P=0.01)$.

\section{Discussion}

The present study examined the performance of subjects that had been taking BZ medications chronically for an average of 5 years on a series of objective behavioral tests 
and subjective mood scales. The performance of the longterm BZ users was compared with age-matched drug-free subjects diagnosed with an anxiety disorder. Few behavioral differences were found between the two groups of subjects. Specifically, the CFF threshold was significantly lower and the subjective report of tranquilization was greater in the chronically medicated subjects. Since the long-term BZ users maintained use of their medication in order to control anxiety symptoms, the group of drug-free anxious subjects probably provided as close an estimate of these subject's behavior prior to their initiating medical treatment as possible to obtain. Furthermore, the use of an anxious control group allowed us to measure the increased feelings of tranquilization in the chronic BZ users, whch agreed with the report from $75 \%$ of the chronically-medicated subjects that continued use of their medication helped to control their anxiety symptoms. Although it is possible that different BZs may cause different profiles of behavioral impairment or that negative results may be due to an excessively heterogenous group of subjects, our attempt to evaluate the BZ medications on the basis of pharmacological half-life did not reveal fundamental differences in their effects.

These results do not support the idea that serious psychomotor or cognitive impairment is necessarily produced in patients by the regular long-term use of BZs (Bergman et al. 1980; Hendler et al. 1980), at least when the medications are taken at medically-prescribed doses. Recently, patients taking BZs chronically demonstrated reductions in performance on the DSST and symbol copying test compared with a group of normal controls, and their performance failed to improve to normal values even when their medication was stopped (Petursson et al. 1983). One problem in evaluating the behavioral status of the chronic drug users is the difficulty in estimating what the subject's performance might have been prior to, or without, drug treatment. Without having assessed the performance of anxious subjects prior to medical treatment, the behavioral deficits shown by patients after chronic BZ medication by Petursson et al. (1983) either may have been already present prior to treatment, or might be long-lasting behavioral changes caused by chronic BZ use. Nevertheless, comparable behavioral deficits on the DSST or symbol copying test or further differences in behavioral performance, beyond reduced CFF threshold and increased tranquilization, were not measured in long-term users of $\mathrm{BZ}$ medications in the present study.

The behavioral effects produced acutely following the initial administration of BZs to normal volunteers have been reported frequently (for review, see Vogel 1979; Wittenborn 1979; Kleinknecht and Donaldson 1975), but similar effects have not been studied previously in long-term users. The assessment of acute effects of BZs in chronicallymedicated subjects could reveal changes in their behavioral effects following long-term use. In addition, the time following the previous dose of medication would be expected to be important for assessing the behavioral effects of BZs, but clinical studies often have minimal control over this variable. For these reasons, the performance of the chronically-medicated subjects was measured $1 \mathrm{~h}$ after drug intake and was compared with test scores measured earlier just prior to administration of the drugs. Several behavior measures changed acutely after BZ intake. Performance improved on the DSST, the CFF threshold decreased, delayed recall was impaired, and the subjective report of tranquiliza- tion was increased whereas physical sedation was decreased. Although different BZs could demonstrate individual profiles of behavioral effects, the BZ medications examined in the present study were not different in the effects they caused in the chronic users.

Taken together with their behavioral performance at the baseline examination, the sensitivity of the behavior tasks to BZs in chronically medicated subjects could be heuristically classified into three broad categories: 1) behavior changes that may be relatively long lasting or continuously present; 2) behavior effects that were present only shortly after drug intake; and 3) behaviors reported to be altered by BZs in drug-free subjects but that were not changed in the chronic BZ users, possibly due to the development of tolerance.

First, the reduction of CFF thresholds and the increased subjective feeling of tranquilization appeared to be more continuously affected by long-term BZ intake. Each of these behaviors were altered during the baseline examination, 4-14 $\mathrm{h}$ following the previous drug dose, as well as changed shortly after taking the $\mathrm{BZ}$ medications. Although both behaviors are caused by acute administration of BZs in drug-free subjects, the chronic users showed these behavior effects in the absence of accompanying subjective feelings of sedation or other impairments in psychomotor performance that are frequently also reported in drug-free subjects. That chronically medicated subjects show such behavior changes suggests that complete tolerance does not develop to these behavioral effects of BZs, and their relative persistence may be a function of accumulated plasma BZ concentrations.

A second type of behavioral response in the chronic users was measured on memory function. The delayed recall of word lists was impaired in chronic users when measured shortly after taking medication, but was not altered at the baseline examination which occurred hours following the previous dose. Thus, memory impairment appeared to occur for only a brief time in the chronic users following intake of their medication, but was not continuously altered despite the persistent concentrations of BZs measured in plasma. A number of BZ medications have been reported to produce effects resembling short-term anterograde amnesia when administered acutely to normal volunteers (Ghoneim and Mewaldt 1975) or to anxious subjects (Angus and Romney 1984; Hartley et al. 1982). These effects appear to be due to an inability to consolidate new information into long-term memory storage, although immediate memory and information learned prior to drug administration are not affected by acute BZ administration (for review, see Lister 1985). In a previous study using normal subjects, Ghoneim et al. (1981) reported that diazepam impaired verbal recall after 3 weeks of daily drug administration but noted some development of tolerance. When subjects were tested 12-14 h after their previous dose of medication, however, inhibition of verbal recall was small but marginally significant. Another study involving short-term chronic administration of diazepam failed to measure memory impairment several hours following the subject's previous dose (Ghoneim et al. 1984a). A similar lack of correlation between the time course of memory impairment and the expected duration of plasma BZ levels has been noted in previous studies with drug-free subjects (Ghoneim et al. 1984b; Kothary et al. 1981; File and Bond 1979). We did not have the opportunity to determine whether the time course of 
memory impairment was altered by chronic BZ use. The results from the present study also suggest that the time course for reduction of the CFF threshold and increased tranquilization after acute BZ administration would differ from the time course of memory impairment.

The effect of BZ medications on memory function has potential implications for the safety of the clinical long-term use of BZs. Although complete tolerance to BZ's effects on verbal recall failed to develop in the chronic users, the effects appeared to be relatively brief in duration. Since the memory-impairing effects of BZs would be limited only to new information presented during a short critical period of time, one may question whether patients taking BZ medications chronically would suffer from or be aware of any effects on their memory. The recall of information learned prior to medication may be facilitated by diazepam (Hinrichs et al. 1984). On the other hand, many patients take BZ medications several times daily, and the memory-impairing effect of BZs may have greater impact for certain kinds of patients, such as cardiac patients (Frazure-Smith and Rolicz-Woloszyk 1982) or the elderly (Salzman et al. 1975). The implications of laboratory tests of recall ability with BZs for the overall clinical status of patients' memory function should be evaluated with further clinical studies.

The third class of behavioral response to chronic $\mathrm{BZ}$ administration is behavioral effects that develop tolerance following the continued use of $\mathrm{BZ}$ medications. Tolerance can be defined as the diminished effects on task performance of $\mathrm{BZs}$ following continued administration. In the present study, the chronic BZ users did not demonstrate significant impairment on the DSST, symbol copying, letter cancellation, nor report significant mental or physical sedation at their baseline examination or following the acute administration of $\mathrm{BZ}$ medications. The present study is limited in concluding that tolerance developed to the effects of BZs on these behaviors because the effects of similar doses of BZs were not examined in drug-free subjects, although further studies are planned to do this. The suggestion that tolerance develops to the effects of BZs on at least some of these behaviors can be made, however, in view of the extensive previous literature concerning BZ's behavioral effects (Vogel 1979; Wittenborn 1979; Kleinknecht and Donaldson 1975), and because some acute behavioral effects of BZs were measured in the chronic users. The lack of psychomotor impairment caused by these doses must also be viewed in consideration of the presence of high plasma levels of BZs and metabolites in these subjects. Although the low doses of some of the BZ medications may not have been expected to cause large impairment of these behaviors, these doses are commonly recommended for medical treatment and indicate that the chronic users probably do not experience such behavioral impairment as a function of their clinical use. The finding that performance on many of the behavior tests used in the present study was not impaired following the chronic use of $\mathrm{BZ}$ medications is in agreement with studies that have demonstrated tolerance following the regular use of BZs for just a few weeks in normal volunteers with similar tasks (Aranko et al. 1983; Liljequist et al. 1979). The lack of impairment found on the DSST and symbol copying tests in the present study is especially significant, because Petursson et al. (1983) reported that these behaviors were impaired in chronic $\mathrm{BZ}$ users. If the use of $\mathrm{BZ}$ medications was responsible for such effects, then DSST and symbol copying performance should be diminished further acutely following
BZ intake in chronic users. This expected result was not obtained in the present study.

Several subjects were also examined during withdrawal from BZ medications and their performance was compared with their baseline performance at the initial interview. During withdrawal, the CFF threshold was increased and the subjective report of tranquilization was reduced while physical sedation was increased. The change in CFF threshold and degree of tranquilizaton occurred in the opposite direction of that measured during the initial interview. The increased anxiety reported by these patients could have been symptomatic of withdrawal caused by the abrupt discontinuation of long-term BZ use (Schopf 1983; Winokur and Rickels 1981; Winokur et al. 1980), or due to a return of anxiety symptoms that caused the patients to originally seek treatment (Rickels et al. 1983; Rickels 1981). The increased $\mathrm{CFF}$ threshold during $\mathrm{BZ}$ withdrawal may reflect a rebound from the continuous CNS depression present during chronic use of BZ medications. The increased report of sedation may have been caused by a lack of sleep for some subjects after suddenly stopping regular BZ use, but this was not accompanied by impaired performance on the other behavior tasks.

Although learning effects could influence the results of psychomotor tests that are readministered frequently, the effects of practice in the present study would be minimal given the lengthy interval between test sessions (unpublished data). Retesting effects would exert more of a role during examination of the acute effects of BZs because the test battery was administered twice within hours, and may have caused the significant increase in DSST performance in the chronic BZ users. Further studies are planned to compare the acute behavioral effects of $\mathrm{BZs}$ in drug-free and chronically-medicated subjects with placebo-controlled studies.

That different behaviors show different types of responses to the long-term use of BZ medications has important implications for the safety of their clinical use as well as the neuropharmacological substrates underlying their effects. That tolerance fails to develop rapidly to the tranquilizing actions of BZs in most patients, but does develop to their motor-impairing and sedative actions, has probably enabled BZs to be used chronically for the clinical treatment of anxiety disorders with relative safety (Rickels et al. 1983; Rickels 1978). However, the clinical significance of the lack of tolerance to the memory-impairing effects of BZs should be examined further and long-term use of even therapeutic doses may cause physical dependence in some patients (Schopf 1983; Rickels et al. 1983; Winokur and Rickels 1981). That tolerance develops selectively to the different behavioral effects of $\mathrm{BZs}$ may indicate differential adaptation of $\mathrm{BZ}$ receptors or other neurochemical substrates modulating these behavioral effects (Lippa et al. 1982). The examination of the behavioral effects of BZs in chronically-medicated subjects provides an important evaluation of their safety as well as clues to the pharmacological mechanisms underlying their beneficial clinical effects.

Acknowledgements. This research was supported by USPHS grant MH-08957. We are very grateful to Dr. David Greenblatt for the analysis of plasma samples for benzodiazepines and their metabolites. We also thank Dr. George Case, Dr. Andrew Winokur, Dr. Charlotte Swenson, Mrs. Reita Fridman, and Ms. Debra Toogood for their help with various parts of this study. 


\section{References}

Angus WR, Romney DM (1984) The effect of diazepam on patient's memory. J Clin Psychopharmacol 4:203-206

Aranko K, Mattila MJ, Seppala T (1983) Development of tolerance and cross-tolerance to the psychomotor actions of lorazepam and diazepam in man. Br J Clin Pharmacol 15:545-552

Bergman H, Borg S, Holm L (1980) Neuropsychological impairment and exclusive abuse of sedatives or hypnotics. Am J Psychiatry 137:215-217

Cohen S (1981) A clinical appraisal of diazepam. Psychosomatics 22:761-769

de Gier JJ, Hart BJ, Nelemans FA, Bergman H (1981) Psychomotor performance and real driving performance of outpatients receiving diazepam. Psychopharmacology $73: 340-344$

File SE, Bond AJ (1979) Impaired performance and sedation after a single dose of lorazepam. Psychopharmacology 66:309-313

Frazure-Smith N, Rolicz-Woloszyk E (1982) Memory problems after ischemic heart disease episodes: Effects of stress, benzodiazepines and smoking. J Psychosom Res 26:613-622

Ghoneim MM, Mewaldt SP (1975) Effects of diazepam and scopolamine on storage, retrieval and organizational processes in memory. Psychopharmacologia 44:257-262

Ghoneim MM, Mewaldt SP, Berie JL, Hinrichs JV (1981) Memory and performance effects of single and 3-week administration of diazepam. Psychopharmacology 73:147-151

Ghoneim MM, Hinrichs JV, Mewaldt SP (1984a) Dose-response analysis of the behavioral effects of diazepam: I. Learning and memory. Psychopharmacology 82:291-295

Ghoneim MM, Hinrichs JV, Noyes R, Anderson DJ (1984b) Behavioral effects of diazepam and propranolol in patients with panic disorder and agoraphobia. Neuropsychobiology $11: 229-235$

Hartley LR, Spencer J, Williamson J (1982) Anxiety, diazepam and retrieval from semantic memory. Psychopharmacology $76: 291-293$

Hendler N, Cimini C, Terrence MA, Long D (1980) A comparison of cognitive impairment due to benzodiazepines and to narcotics. Am J Psychiatry 137:828-830

Hinrichs JV, Ghoneim MM, Mewaldt SP (1984) Diazepam and memory: retrograde facilitation produced by interference reduction. Psychopharmacology 84:158-162

Kleinknecht RA, Donaldson D (1975) A review of the effects of diazepam on cognitive and psychomotor performance. J Nerv Ment Dis 161:399-411

Kothary SP, Brown ACD, Pandit UA, Samara SK, Pandit SK (1981) Time course of antirecall effect of diazepam and lorazepam following oral administration. Anesthesiology 55:641-644

Liljequist R, Palva E, Linnoila M (1979) Effects of learning and memory of 2-week treatments with chlordiazepoxide lactam, $\mathrm{N}$-desmethyldiazepam, oxazepam and methyloxazepam, alone or in combination with alcohol. Int Pharmacopsychiatry 14:190-198

Linnoila M, Erwin CW, Brendle A, Simpson D (1983) Psychomotor effects of diazepam in anxious patients and healthy volunteers. J Clin Psychopharmacol 3:88-96

Lippa AS, meyerson LR, Beer B (1982) Molecular substrates of anxiety: Clues from the heterogeneity of the benzodiazepine receptor. Life Sci 31:1409-1417

Lister RG (1985) The amnesic action of benzodiazepines in man Neurosci Biobehav Rev 9:87-94

Lucki I, Rickels K, Geller AM (1985) Psychomotor performance following the long-term use of benzodiazepines. Psychopharmacol Bull 21:93-96

Mellinger GD, Balter MB (1981) Prevalence and patterns of use of psychotherapeutic drugs: Results from a 1979 national survey of American adults. In: Tognoni G, Bellantuono C, Lader $M$ (eds) Epidemiological impact of psychotropic drugs, New York: Elsevier-North Holland, Inc, pp 117-135

Norris H (1971) The action of sedatives on brain stem oculomotor systems in man. Neuropharmacology 10:181-191

Paivio A, Yuille JC, Madigan SA (1968) Concreteness, imagery and meaningfulness values for 925 nouns. J Exp Psychol [Suppl] 76:1-25

Petursson H, Gudjonsson GH, Lader MH (1983) Psychometric performance during withdrawal from long-term benzodiazepine treatment. Psychopharmacology $81: 345-349$

Rickels K (1978) Use of antianxiety agents in anxious outpatients. Psychopharmacology 58:1-17

Rickels K (1981) Benzodiazepines: Use and misuse. In: Klein DF and Rabkin J (eds) Anxiety: New research and changing concepts, New York: Raven Press, pp 1-26

Rickels K, Case GW, Downing RW, Winokur A (1983) Long-term diazepam therapy and clinical outcome. JAMA 250:767-771

Salzman C, Shader RI, Harmantz J, Robertson L (1975) Psychopharmacologic investigations in elderly volunteers: Effect of diazepam in males. J Am Geriatr Soc 23:451-457

Schopf J (1983) Withdrawal phenomena after long-term administration of benzodiazepines: A review of recent investigations. Pharmacopsychiatria 16:1-8

Smith JM, Misiak H (1976) Critical flicker frequency (CFF) and psychotropic drugs in normal subjects - a review. Psychopharmacology 47:175-182

Thorndike EL, Lorge L (1944) The teachers word book of 30,000 words. New York: Columbia University Press

Vogel JR (1979) Objective measurement of human performance changes produced by antianxiety drugs. In: Fielding S, Lal H (eds) Anxiolytics, New York: Futura Publishing Co pp 343374

Wechlser D (1955) Manual for the Wechsler Adult Intelligence Scale. New York: The Psychological Corp

Winokur A, Rickels K (1981) Withdrawal and pseudowithdrawal from diazepam therapy. J Clin Psychiatry 42:442-444

Winokur A, Rickels K, Greenblatt DJ, Snyder P, Schatz NJ (1980) Withdrawal reaction from long-term, low-dosage administration of diazepam. Arch Gen Psychiatry 37:101-105

Wittenborn JR (1970) Effects of benzodiazepines on psychomotor performance. Br J Clin Pharmacol 7:61S-67S

Zimmerman-Tansella C, Tansella M, Lader M (1978) Psychological performance in anxious patients treated with diazepam. Prog Neuro-Psychopharmacol 3:361-368

Received April 25, 1985 